\title{
Frutanos do tipo inulina e aumento da absorção de cálcio: uma revisão sistemática ${ }^{1}$
}

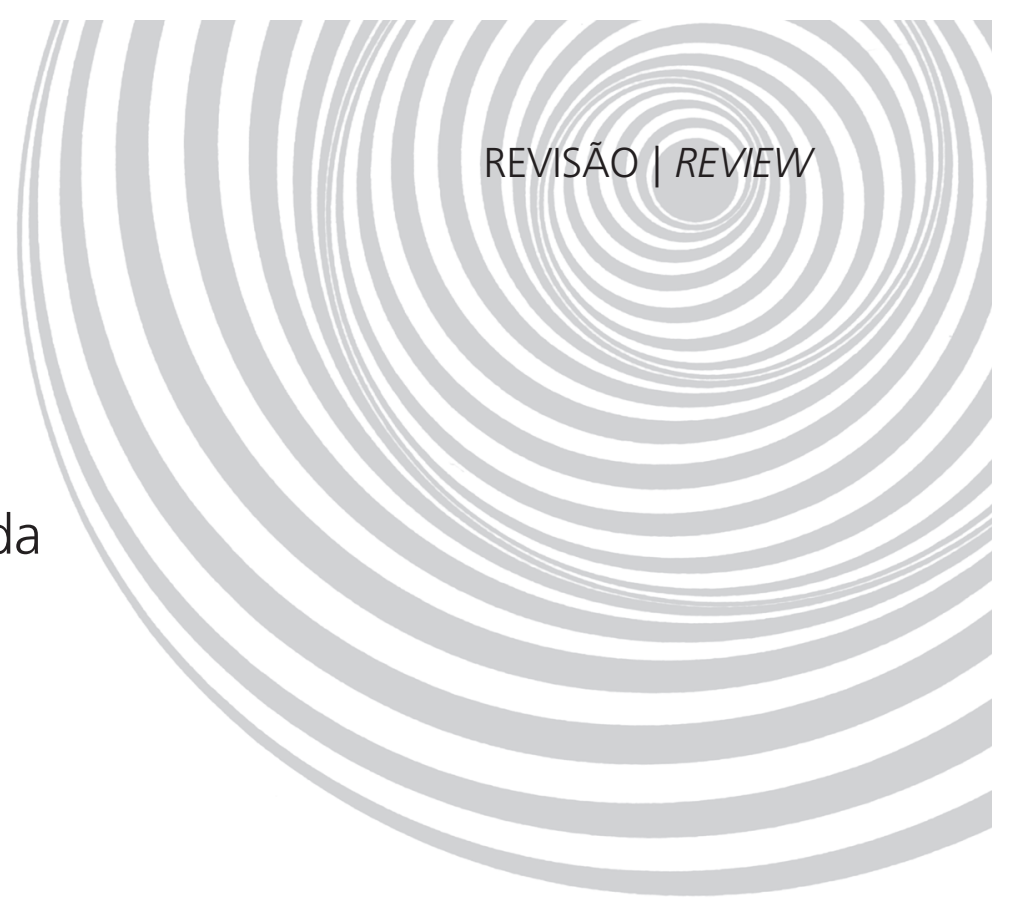

\section{Inulin-type fructans and calcium absorption enhancement:} a systematic review

Vanessa Dias CAPRILES 2

José Alfredo Gomes ARÊAS

RE S U M O

Realizou-se uma revisão sistemática da literatura sobre os efeitos do consumo de frutanos do tipo inulina na absorção de cálcio. Resultados de quatro dos sete ensaios clínicos duplo-cegos controlados aleatorizados relatados neste trabalho indicam que o consumo diário da mistura de frutanos (inulina e oligofrutose 1:1) aumenta a absorção de cálcio, dependendo da idade cronológica e fisiológica, bem como do estado menopausal de indivíduos saudáveis que consomem quantidades adequadas de cálcio. O número limitado de ensaios clínicos realizados e as diferenças relativas ao planejamento experimental, tempo de estudo, tipo e quantidade de frutano consumido (inulina, oligofrutose ou a mistura de ambos), bem como a faixa etária dos indivíduos, impedem a generalização dos resultados observados. Esta revisão demonstra a necessidade de mais ensaios clínicos de longa duração, nos quais tanto a absorção de cálcio como a densidade mineral óssea sejam avaliadas. Futuros estudos devem contribuir para a compreensão dos mecanismos de ação dos frutanos no aumento da absorção de cálcio; para avaliar se esse efeito persiste em longo prazo e se pode ser considerado como benefício real para a saúde óssea; e para testar se tais efeitos poderiam beneficiar indivíduos de outras faixas etárias e diferentes condições fisiológicas. Evidências científicas consistentes e acumuladas ainda são necessárias para poder considerar o consumo de frutanos como uma estratégia de prevenção da osteoporose.

Termos de indexação: Cálcio. Densidade óssea. Frutanos. Inulina.

A B S T R A C T

We performed a systematic review of literature regarding the effects of inulin-type fructans consumption on calcium absorption. Results of four from seven double-blind randomized controlled clinical trials revised in this

\footnotetext{
1 Artigo elaborado a partir da tese de VD CAPRILES, intitulada "Otimização de propriedades nutricionais e sensoriais de produtos à base de amaranto enriquecidos com frutanos, para intervenção em celíacos". Universidade de São Paulo; 2009.

2 Universidade Federal de São Paulo, Departamento de Biociências. Campus Baixada Santista, Av. D. Ana Costa, 95, Vl. Mathias, 11060-001, Santos, SP, Brasil. Correspondência para/Correspondence to: VD CAPRILES.E-mail: <vanessa.capriles@unifesp.br>.

${ }^{3}$ Universidade de São Paulo, Departamento de Nutrição, Faculdade de Saúde Pública. São Paulo, SP, Brasil.
} 
148 | VD CAPRILES \& JAG AREAS

work showed that daily consumption of fructans mixture (inulin and oligofructose 1:1) increases calcium absorption, depending on the chronological and physiological age, and menopausal status of healthy individuals who consume adequate amounts of calcium. Due to the limited number of clinical trials and the differences on the experimental design, follow-up, fructans type (inulin, oligofructose or their mixture) and quantity consumed, and the individuals' age, the results cannot be generalized. This review demonstrates the need for more long-term follow-up clinical trial, in which both calcium absorption and bone mineral density are measured. Further studies may help understand the mechanisms underlying the effects of inulin-type fructans on calcium absorption, and to evaluate if the increased calcium absorption is long-term persistence and if it can be translated into real benefits to bone health; and also to test whether these effects could benefit individuals with other age groups and in different physiological conditions. Consistent accumulated scientific evidences are still necessary to consider inulin-type fructans consumption as a prevention strategy for osteoporosis.

Indexing terms: Calcium. Bone density. Fructans. Inulin.

\section{N T R O D U Ç Ã O}

Os frutanos do tipo inulina são carboidratos de reserva encontrados em alimentos comumente consumidos, como banana, alho, cebola e trigo, assim como na raiz da chicória, de onde são extraídos em escala industrial. Estruturalmente, são polímeros lineares de $\beta$-D-frutofuranoses unidas por ligações do tipo $\beta(2 \rightarrow 1)$, que podem estar ligados a uma molécula terminal de $\beta$-D-glucopiranosil, e diferem quanto ao grau de polimerização, sendo que o da oligofrutose é inferior a dez e o da inulina pode variar de onze até sessenta'.

A inulina (grau de polimerização - $\mathrm{GP}_{\text {médio }}=$ 12) é obtida industrialmente a partir da extração com água quente das raízes da chicória (Cichorium intybus), seguida de refinação e secagem por atomização. Três tipos de produtos comerciais podem ser elaborados: a inulina com alto GP $\left(G P_{\text {médio }}=25\right)$, obtida a partir de separação física; a oligofrutose $\left(G P_{\text {médio }}=4\right)$, obtida por meio da hidrólise enzimática parcial da inulina; e a mistura de inulina e oligofrutose, obtida a partir da combinação de $50 \%$ de inulina de alto GP e de $50 \%$ de oligofrutose?

Esses frutanos estão disponíveis comercialmente como ingredientes alimentícios em forma de pó ou xarope, e possuem aplicação na formulação de diferentes tipos de produtos, buscando tanto a sua alegação como alimento funcional, quanto a melhoria de seu valor nutritivo e de suas propriedades sensoriais ${ }^{3}$.
As enzimas intestinais humanas não são capazes de hidrolisar as ligações do tipo $\beta(2 \rightarrow 1)$ encontradas na inulina e na oligofrutose; assim, esses polímeros chegam intactos ao cólon. Como consequência, ocorre o aumento do volume das fezes e da frequência de evacuações. Por conta desses efeitos fisiológicos, são incluídos na categoria de fibras alimentares. No entanto, devido a suas propriedades fermentativas específicas, esses frutanos apresentam efeitos gastrointestinais e sistêmicos distintos das demais fibras².

Os frutanos inulina e oligofrutose são os carboidratos não-disponíveis mais investigados em estudos envolvendo seres humanos, sendo os únicos para os quais foi cientificamente comprovado serem resistentes à acidez gástrica, à hidrólise pelas enzimas gastrointestinais dos mamíferos e à absorção gastrointestinal; serem fermentados pela microbiota intestinal; e estimularem seletivamente o crescimento e/ou a atividade de bactérias intestinais associadas à saúde e bem-estar. Desse modo, satisfazem os três critérios necessários para sua classificação como ingrediente alimentar prebiótico ${ }^{4}$. "Prebiótico é um ingrediente fermentado seletivamente que ocasiona mudanças específicas na composição e/ou atividade da microbiota intestinal, ocasionando benefícios à saúde e ao bem-estar do hospedeiro" 4 .

De acordo com Kolida \& Gibson ${ }^{5}$, o consumo diário de cinco a oito gramas de frutanos parece ser suficiente para se obter o efeito prebiótico, evitando-se efeitos colaterais indesejados como flatulência e desarranjos intestinais consequentes da ingestão de maior quantidade desses compostos. 
Os resultados de algumas pesquisas sugerem que a inulina e a oligofrutose também podem contribuir para a redução dos níveis de triglicérides e LDL-colesterol sérico, a estimulação do sistema imune, a diminuição do risco de doenças colônicas e o aumento da absorção de cálcio².

O cálcio dietético é absorvido por transporte ativo e por difusão passiva. A absorção por transporte ativo (via transcelular, com absorção através das células intestinais) ocorre principalmente na porção inicial do jejuno, por meio da proteína ligadora de cálcio dependente da vitamina D, a calbindina-D9k. Enquanto a absorção por difusão passiva (via paracelular, com absorção entre as células intestinais) ocorre por toda a extensão do intestino delgado e grosso, esse mecanismo é dependente apenas do gradiente de concentração de cálcio entre o lúmen intestinal e os fluidos corporais ${ }^{6}$.

Como resultado da fermentação colônica dos frutanos, há produção de ácidos graxos de cadeia curta (acetato, propionato e butirato) e outros ácidos orgânicos (como o lactato), bem como a produção de gases, acompanhada pelo aumento do conteúdo cecal e pela redução do $\mathrm{pH}$ do lúmen intestinal, o que favorece a solubilidade e a difusão do cálcio através da mucosa colônica. A fermentação induz alterações estruturais na mucosa intestinal, uma vez que o butirato, a principal fonte de energia para a mucosa colônica, estimula a proliferação celular (hipertrofia do (eco), aumentando a área absortiva do epitélio intestinal. Relatos indicam que o butirato estimula a expressão da calbindina-D9k, podendo também influenciar a absorção ativa do cálcio ${ }^{6,7}$.

Resultados de estudos realizados na última década vêm mostrando que o consumo de inulina e oligofrutose aumenta a absorção de cálcio e a densidade mineral óssea de ratos em crescimento, bem como alivia a perda de massa óssea de ratas ovariectomizadas, modelo utilizado para simular a pós-menopausa ${ }^{6,7}$.

Estudos indicam que, devido a seu menor peso molecular, a oligofrutose é fermentada mais rapidamente, no cólon proximal, enquanto a inulina é fermentada mais lentamente nas partes distais do cólon. Sugere-se que a combinação dos dois tipos de frutanos exerce efeito sinergista na absorção de cálcio, uma vez que a fermentação e a hipertrofia celular são estimuladas por toda a extensão do cólon, aumentando, portanto, a área por onde o cálcio pode ser absorvido ${ }^{8,9}$. Os resultados promissores observados em modelos animais estimularam a avaliação do efeito dos frutanos na biodisponibilidade de cálcio em seres humanos.

O presente artigo de revisão descreve os resultados de ensaios clínicos duplo-cegos controlados aleatorizados, para a avaliação dos efeitos do consumo de frutanos do tipo inulina na absorção intestinal de cálcio de indivíduos saudáveis.

\section{MÉTODOS}

Para a seleção dos artigos adotou-se a técnica de revisão sistemática, com o objetivo de identificar ensaios clínicos duplo-cegos controlados aleatorizados, para a avaliação do consumo de frutanos do tipo inulina (inulina, oligofrutose e/ou a mistura de ambos) na absorção de cálcio e na densidade mineral óssea de indivíduos saudáveis.

Por se tratar de uma revisão de literatura, o estudo não foi submetido ao Comitê de Ética em Pesquisa da instituição.

Foram incluídos estudos originais e publicados, do tipo ensaio clínico, conduzidos em indivíduos saudáveis. Os artigos relatavam: (1) alocação aleatória dos grupos; (2) pelo menos um grupo em tratamento e um grupo de controle; (3) existência de duplo-cego; (4) avaliação da absorção de cálcio e/ou da densidade mineral óssea.

Foram excluídos os estudos conduzidos em indivíduos: (1) com diagnóstico prévio de doença intestinal, renal e/ou relacionada à homeostase de cálcio e à saúde óssea; (2) em uso de drogas, suplementos nutricionais e compostos bioativos que alteram a absorção de cálcio; (3) em uso de terapia de reposição hormonal; (4) recém-nascidos, gestantes e nutrizes. Foram excluídas 
as publicações que apresentavam informações repetidas ou disponíveis em outros artigos.

A pesquisa dos artigos foi realizada em todas as bases de dados do Institute for Scientific Information (ISI), por meio do ISI Web of Knowledge.

Foram utilizadas as seguintes palavras-chave: frutanos (fructans), frutanos do tipo inulina (inulin-type fructans), inulina (inulin) e oligofrutose (oligofructose), associadas aos termos cálcio (calcium), osso (bone) e densidade mineral óssea (bone mineral density). A busca limitou-se aos artigos escritos em português, espanhol e inglês, com data-limite de publicação até dezembro de 2009.

Os artigos rejeitados na primeira triagem, a partir da análise do título e do resumo, apresentavam inadequação aos critérios de inclusão ou presença de algum dos critérios de exclusão da revisão. Nos casos de incerteza ou discordância, o texto completo do artigo era consultado para confirmar sua elegibilidade. Em complementação à busca nas bases de dados, as listas de referências bibliográficas dos artigos incluídos foram consultadas para identificar algum possível estudo relevante não identificado anteriormente.

Os artigos foram sistematicamente revisados em relação ao consumo de frutanos, delineamento do estudo, presença de cointervenção, método de avaliação da absorção de cálcio, variáveis dietéticas e metabólicas exploradas e resultados encontrados. A absorção de cálcio foi adotada como o desfecho principal, sendo consideradas significativas as diferenças apuradas com valor de $p<0,05$.

Em decorrência da heterogeneidade das características dos indivíduos, dos delineamentos dos estudos, do tipo e quantidade de frutanos consumidos e do método de avaliação da absorção de cálcio, não foi possível realizar a síntese por metanálise.

\section{RES U LTA D OS}

Dos setenta e oito artigos identificados mediante as palavras-chave empregadas, sete estu- dos publicados atendiam a todos os critérios de inclusão estabelecidos.

Os sete artigos selecionados foram sintetizados (Quadro 1) segundo a faixa etária dos indivíduos (adolescentes, adultos jovens e mulheres na pós-menopausa). Os estudos foram desenvolvidos em países europeus (Holanda ${ }^{10,11}$ e França ${ }^{12}$ ) e nos Estados Unidos da América ${ }^{13-16}$, com os protocolos experimentais aprovados pelos Comitês de Ética em Pesquisa das respectivas instituições. Os participantes e/ou seus responsáveis legais foram esclarecidos a respeito dos objetivos e procedimentos adotados e consentiram em participar dos estudos.

\section{Caracterização dos indivíduos e cálculo do tamanho da amostra}

Observou-se que os indivíduos selecionados nos estudos relatados neste trabalho apresentavam peso adequado para a idade ou índice de massa corpórea na faixa da normalidade, e foram considerados saudáveis após a avaliação de seu estado de saúde por meio de exame físico, clínico e histórico médico.

O efeito do consumo de frutanos em adolescentes foi avaliado em quatro estudos. van den Heuvel et al. ${ }^{11}$ avaliaram somente meninos, Griffin et al. ${ }^{13,14}$ recrutaram apenas meninas pré-púberes e púberes (com idade próxima à primeira menarca), e Abrams et al. ${ }^{15}$ selecionaram meninos e meninas pré-púberes.

De acordo com os critérios pré-estabelecidos, apenas um estudo com adultos jovens foi encontrado ${ }^{10}$. Foram localizados dois estudos com mulheres na pós-menopausa. Tahiri et al. ${ }^{12}$ selecionaram mulheres que estavam há pelo menos dois anos na pós-menopausa, enquanto Holloway et al. ${ }^{16}$ recrutaram mulheres que estavam há pelo menos uma década nessa fase.

O cálculo do tamanho da amostra foi relatado apenas nos estudos realizados com adolescentes. Para tanto, os autores utilizaram dados previamente publicados na literatura e consideraram um poder de $90 \%$ para detectar 
Quadro 1. Descrição dos estudos incluídos: efeitos do consumo de frutanos do tipo inulina na absorção de cálcio de indivíduos saudáveis.

\begin{tabular}{|c|c|c|c|c|c|c|}
\hline \multirow[b]{2}{*}{$\begin{array}{l}\text { Tipo de frutano e } \\
\text { quantidade consumida } \\
\text { (g/dia) }\end{array}$} & \multirow[b]{2}{*}{$\begin{array}{l}\text { Caracterização } \\
\text { dos indivíduos }\end{array}$} & \multirow[b]{2}{*}{$\begin{array}{l}\text { Delineamento } \\
\text { do estudo e } \\
\text { tipo de controle }\end{array}$} & \multirow[b]{2}{*}{$\begin{array}{l}\text { Tempo de } \\
\text { seguimento }\end{array}$} & \multicolumn{2}{|c|}{ Absorção de cálcio } & \multirow[b]{2}{*}{ Referência } \\
\hline & & & & $\begin{array}{l}\text { Método } \\
\text { utilizado }\end{array}$ & $\begin{array}{l}\text { Resultados e } \\
\text { estatística }\end{array}$ & \\
\hline \multicolumn{7}{|c|}{ Adolescentes } \\
\hline $\begin{array}{l}\text { Oligofrutose (15g/dia, } \\
3 \text { doses de } 5 \mathrm{~g} \text { ) } \\
\text { (Raftilose P95, Orafti) }\end{array}$ & $\begin{array}{l}12 \text { meninos } \\
\text { (14-16 anos) } \\
\text { Consumo de } \\
\text { cálcio não foi } \\
\text { avaliado. }\end{array}$ & $\begin{array}{l}\text { Ensaio } \\
\text { aleatorizado } \\
\text { cruzado, duplo- } \\
\text {-cego, placebo- } \\
\text {-controlado } \\
\text { (sacarose) }\end{array}$ & $\begin{array}{l}9 \text { dias de } \\
\text { intervenção, } \\
19 \text { dias de } \\
\text { washout }\end{array}$ & $\begin{array}{l}\text { Recuperação } \\
\text { relativa de } \\
{ }^{44} \mathrm{Ca} \mathrm{e} \mathrm{e}{ }^{46} \mathrm{Ca} \\
\text { na urina de } \\
36 \text { horas }\end{array}$ & $\begin{array}{l}\text { Maior no grupo tratamento } \\
(\mathrm{M}=60,1, \mathrm{DP}=17,2 \%) \text { em } \\
\text { relação ao controle } \\
(\mathrm{M}=47,8, \mathrm{DP}=16,4 \%) \\
p<0,05 \\
\text { Teste } t \text { pareado }\end{array}$ & $\begin{array}{l}\text { Van den } \\
\text { Heuvel et al. }{ }^{11}\end{array}$ \\
\hline $\begin{array}{l}\text { Oligofrutose ( } 8 \mathrm{~g} / \mathrm{dia} \text {, } \\
2 \text { doses de } 4 \mathrm{~g})\end{array}$ & $\begin{array}{l}30 \text { meninas } \\
\text { (11-14 anos) } \\
\text { Consumo de } \\
\text { cálcio: } \\
\sim 1500 \mathrm{mg} / \mathrm{dia}\end{array}$ & $\begin{array}{l}\text { Ensaio } \\
\text { aleatorizado } \\
\text { cruzado, duplo- } \\
\text {-cego, placebo- } \\
\text {-controlado } \\
\text { (sacarose) }\end{array}$ & $\begin{array}{l}21 \text { dias de } \\
\text { intervenção, } \\
14 \text { dias de } \\
\text { washout }\end{array}$ & $\begin{array}{l}\text { Recuperação } \\
\text { relativa de } \\
{ }^{46} \mathrm{Ca} \text { e }{ }^{42} \mathrm{Ca} \\
\text { na urina de } \\
48 \text { horas }\end{array}$ & $\begin{array}{l}\text { Não houve diferença entre } \\
\text { os grupos tratamento } \\
(\mathrm{M}=31,8, \mathrm{DP}=9,3 \%) \text { e } \\
\text { controle }(\mathrm{M}=31,8, \\
\mathrm{DP}=10,0 \%), p=0,75 \\
\text { Teste } t \text { pareado }\end{array}$ & \multirow{2}{*}{ Griffin et al. ${ }^{13}$} \\
\hline $\begin{array}{l}\text { Inulina+Oligofrutose } \\
\text { (8g/dia,2 doses de 4g) } \\
\text { (Synergy1, Orafti) }\end{array}$ & $\begin{array}{l}29 \text { meninas } \\
\text { (11-14 anos) } \\
\text { Consumo de } \\
\text { cálcio: } \\
\sim 1500 \mathrm{mg} / \mathrm{dia}\end{array}$ & $\begin{array}{l}\text { Ensaio } \\
\text { aleatorizado } \\
\text { cruzado, duplo- } \\
\text {-cego, placebo- } \\
\text {-controlado } \\
\text { (sacarose) }\end{array}$ & $\begin{array}{l}21 \text { dias de } \\
\text { intervenção, } \\
14 \text { dias de } \\
\text { washout }\end{array}$ & $\begin{array}{l}\text { Recuperação } \\
\text { relativa de } \\
{ }^{46} \mathrm{Ca} \mathrm{e} \mathrm{e}{ }^{42} \mathrm{Ca} \\
\text { na urina de } \\
48 \text { horas }\end{array}$ & $\begin{array}{l}\text { Maior no grupo tratamento } \\
(\mathrm{M}=38,2, \mathrm{DP}=9,8 \%) \text { em } \\
\text { relação ao controle } \\
(\mathrm{M}=32,3, \mathrm{DP}=9,8 \%), \\
p=0,007 \\
\text { Teste } t \text { pareado }\end{array}$ & \\
\hline $\begin{array}{l}\text { Inulina+Oligofrutose } \\
\text { (8g/dia, } 2 \text { doses de } 4 \mathrm{~g})\end{array}$ & $\begin{array}{l}54 \text { meninas } \\
\text { (10-15 anos) } \\
\text { Consumo de } \\
\text { cálcio: } \\
\text { M=1 390, } \\
\text { DP=453mg/dia }\end{array}$ & $\begin{array}{l}\text { Ensaio } \\
\text { aleatorizado } \\
\text { cruzado, duplo- } \\
\text {-cego, placebo- } \\
\text {-controlado } \\
\text { (sacarose) }\end{array}$ & $\begin{array}{l}21 \text { dias de } \\
\text { intervenção, } \\
14 \text { dias de } \\
\text { washout }\end{array}$ & $\begin{array}{l}\text { Recuperação } \\
\text { relativa de } \\
{ }^{46} \mathrm{Ca} \mathrm{e} \mathrm{e}{ }^{42} \mathrm{Ca} \\
\text { na urina de } \\
48 \text { horas }\end{array}$ & $\begin{array}{l}\text { Maior no grupo tratamento } \\
(\mathrm{M}=36,1, \mathrm{DP}=9,8 \%) \text { em } \\
\text { relação ao controle } \\
(\mathrm{M}=33,1, \mathrm{DP}=9,2 \%), \\
p=0,027 \\
\text { Teste } t \text { pareado }\end{array}$ & Griffin et al. ${ }^{14}$ \\
\hline $\begin{array}{l}\text { Inulina+ Oligofrutose } \\
\text { (8g/dia, } 1 \text { dose de } 8 g \text { ) }\end{array}$ & \begin{tabular}{|l|}
98 meninos e \\
meninas \\
(9-13 anos) \\
48 no grupo \\
tratamento e 50 \\
no controle. \\
Consumo de \\
cálcio: 900 - \\
1 000mg/dia
\end{tabular} & $\begin{array}{l}\text { Ensaio } \\
\text { aleatorizado, } \\
\text { paralelo, duplo- } \\
\text {-cego, placebo- } \\
\text {-controlado } \\
\text { (maltodextrina) }\end{array}$ & $\begin{array}{l}56 \text { dias de } \\
\text { intervenção }\end{array}$ & $\begin{array}{l}\text { Recuperação } \\
\text { relativa de } \\
{ }^{46} \mathrm{Ca} \mathrm{e} \mathrm{e}{ }^{42} \mathrm{Ca} \\
\text { na urina de } \\
48 \text { horas }\end{array}$ & $\begin{array}{l}\text { Maior no grupo tratamento } \\
(\mathrm{M}=38,5, \mathrm{DP}=1,2 \%) \text { em } \\
\text { relação ao controle } \\
(\mathrm{M}=30,0, \mathrm{DP}=1,3 \%) \\
p<0,001 \mathrm{Análise} \text { de } \\
\text { covariância para medidas } \\
\text { repetidas }\end{array}$ & \multirow{2}{*}{ Abrams et al..$^{15}$} \\
\hline $\begin{array}{l}\text { Inulina+ oligofrutose } \\
\text { (8g/dia, } 1 \text { dose de } 8 \mathrm{~g} \text { ) }\end{array}$ & $\begin{array}{l}92 \text { meninos e } \\
\text { meninas } \\
\text { (9-13 anos) } \\
47 \text { no grupo } \\
\text { tratamento e } 45 \\
\text { no controle. } \\
\text { Consumo de } \\
\text { cálcio: } 900 \text { - } \\
1 \text { 000mg/dia }\end{array}$ & $\begin{array}{l}\text { Ensaio } \\
\text { aleatorizado, } \\
\text { paralelo, duplo- } \\
\text {-cego, placebo- } \\
\text {-controlado } \\
\text { (maltodextrina) }\end{array}$ & $\begin{array}{l}365 \text { dias de } \\
\text { intervenção }\end{array}$ & $\begin{array}{l}\text { Recuperação } \\
\text { relativa de } \\
{ }^{46} \mathrm{Ca} \mathrm{e} \mathrm{e}{ }^{42} \mathrm{Ca} \\
\text { na urina de } \\
48 \text { horas }\end{array}$ & $\begin{array}{l}\text { Maior no grupo tratamento } \\
(\mathrm{M}=37,7, \mathrm{DP}=2,1 \%) \text { em } \\
\text { relação ao controle } \\
(\mathrm{M}=31,7, \mathrm{DP}=2,3 \%) \\
p=0,04 \\
\text { Análise de covariância } \\
\text { para medidas repetidas }\end{array}$ & \\
\hline
\end{tabular}


152 | VD CAPRILES \& JAG AREAS

Quadro 1. Descrição dos estudos incluídos: efeitos do consumo de frutanos do tipo inulina na absorção de cálcio de indivíduos saudáveis.

Conclusão

\begin{tabular}{|c|c|c|c|c|c|c|}
\hline \multirow[b]{2}{*}{$\begin{array}{l}\text { Tipo de frutano e } \\
\text { quantidade consumida } \\
\text { (g/dia) }\end{array}$} & \multirow[b]{2}{*}{$\begin{array}{l}\text { Caracterização } \\
\text { dos indivíduos }\end{array}$} & \multirow[b]{2}{*}{$\begin{array}{l}\text { Delineamento } \\
\text { do estudo e } \\
\text { tipo de controle }\end{array}$} & \multirow[b]{2}{*}{$\begin{array}{l}\text { Tempo de } \\
\text { seguimento }\end{array}$} & \multicolumn{2}{|c|}{ Absorção de cálcio } & \multirow[b]{2}{*}{ Referência } \\
\hline & & & & $\begin{array}{l}\text { Método } \\
\text { utilizado }\end{array}$ & $\begin{array}{c}\text { Resultados e } \\
\text { estatística }\end{array}$ & \\
\hline \multicolumn{7}{|c|}{ Adultos jovens } \\
\hline $\begin{array}{l}\text { Oligofrutose (15g/dia, } \\
3 \text { doses de } 5 \mathrm{~g} \text { ) } \\
\text { (não informado) }\end{array}$ & \multirow{2}{*}{$\begin{array}{l}12 \text { homens } \\
\text { (20-30 anos). } \\
\text { Teor de cálcio } \\
\text { dietético: } \\
\text { 955mg/dia }\end{array}$} & \multirow{2}{*}{$\begin{array}{l}\text { Ensaio } \\
\text { aleatorizado } \\
\text { cruzado, duplo- } \\
\text {-cego, com } \\
\text { dieta-controle } \\
\\
\text { Quatro } \\
\text { tratamentos:1) } \\
\text { dieta-controle } \\
+15 g \text { inulina/ } \\
\text { dia 2) dieta- } \\
\text {-controle + 15g } \\
\text { oligofrutose/dia } \\
\text { 3) dieta- } \\
\text {-controle + 15g } \\
\text { galacto- } \\
\text {-oligosacarídeo/ } \\
\text { dia 4) dieta- } \\
\text {-controle }\end{array}$} & $\begin{array}{l}21 \text { dias de } \\
\text { intervenção, } \\
\text { tempo de } \\
\text { washout } \\
\text { não } \\
\text { informado }\end{array}$ & $\begin{array}{l}\text { Recuperação } \\
\text { relativa de } \\
{ }^{44} \mathrm{Ca} \mathrm{e}{ }^{48} \mathrm{Ca} \\
\text { na urina de } \\
24 \text { horas }\end{array}$ & $\begin{array}{l}\text { Não houve diferença entre } \\
\text { os grupos tratamento } \\
(\mathrm{M}=26,3, \mathrm{DP}=1,9 \%) \text { e } \\
\text { controle }(\mathrm{M}=28,1, \\
\mathrm{DP}=4,3 \%), p>0,05 \\
\text { Análise de variância com } \\
\text { blocos aleatorizados }\end{array}$ & \multirow{2}{*}{$\begin{array}{l}\text { Van den } \\
\text { Heuvel et al. }{ }^{10}\end{array}$} \\
\hline $\begin{array}{l}\text { Inulina (15g/dia, } \\
3 \text { doses de } 5 \mathrm{~g} \text { ) } \\
\text { (não informado) }\end{array}$ & & & $\begin{array}{l}21 \text { dias de } \\
\text { intervenção, } \\
\text { tempo de } \\
\text { washout } \\
\text { não } \\
\text { informado }\end{array}$ & $\begin{array}{l}\text { Recuperação } \\
\text { relativa de } \\
{ }^{44} \mathrm{Ca} \mathrm{e}{ }^{48} \mathrm{Ca} \\
\text { na urina de } \\
24 \text { horas }\end{array}$ & $\begin{array}{l}\text { Não houve diferença entre } \\
\text { os grupos tratamento } \\
(\mathrm{M}=25,8, \mathrm{DP}=2,3 \%) \text { e } \\
\text { controle }(\mathrm{M}=28,1 \text {, } \\
\mathrm{DP}=4,3 \%), p>0,05 \\
\text { Análise de variância com } \\
\text { blocos aleatorizados }\end{array}$ & \\
\hline \multicolumn{7}{|c|}{ Mulheres na pós-menopausa } \\
\hline $\begin{array}{l}\text { Oligofrutose (10g/dia, } \\
2 \text { doses de } 5 \mathrm{~g} \text { ) } \\
\text { (não informado) }\end{array}$ & $\begin{array}{l}12 \text { mulheres } \\
\text { (50-70 anos, >2 } \\
\text { anos na } \\
\text { pós-menopausa). } \\
\text { Consumo de } \\
\text { cálcio: } ~ 900 \mathrm{mg} / \\
\text { dia }\end{array}$ & $\begin{array}{l}\text { Ensaio } \\
\text { aleatorizado } \\
\text { cruzado, duplo- } \\
\text {-cego, placebo- } \\
\text {-controlado } \\
\text { (sacarose) }\end{array}$ & $\begin{array}{l}35 \text { dias de } \\
\text { intervenção, } \\
21 \text { dias de } \\
\text { washout }\end{array}$ & $\begin{array}{l}\text { Recuperação } \\
\text { relativa de } \\
{ }^{44} \text { Ca nas fezes } \\
\text { de } 7 \text { dias }\end{array}$ & $\begin{array}{l}\text { Não houve diferença entre } \\
\text { os grupos tratamento } \\
(\mathrm{M}=35,6, \mathrm{DP}=9,4 \%) \text { e } \\
\text { controle }(\mathrm{M}=36,5, \\
\mathrm{DP}=8,5 \%), p>0,05 \\
\text { Teste } t \text { pareado }\end{array}$ & Tahiri et al. ${ }^{12}$ \\
\hline $\begin{array}{l}\text { Inulina+ Oligofrutose } \\
(10 \mathrm{~g} / \mathrm{dia}, 2 \text { doses de } \\
5 \mathrm{~g})\end{array}$ & $\begin{array}{l}15 \text { mulheres } \\
\text { (M=72, DP=6 } \\
\text { anos, >10 anos } \\
\text { na pós- } \\
\text {-menopausa). } \\
\text { Consumo de } \\
\text { cálcio: } \\
\text { 1 000mg/dia }\end{array}$ & $\begin{array}{l}\text { Ensaio } \\
\text { aleatorizado } \\
\text { cruzado, duplo- } \\
\text {-cego, placebo- } \\
\text {-controlado } \\
\text { (maltodextrina) }\end{array}$ & $\begin{array}{l}42 \text { dias de } \\
\text { intervenção, } \\
42 \text { dias de } \\
\text { washout }\end{array}$ & $\begin{array}{l}\text { Recuperação } \\
\text { relativa de } \\
{ }^{46} \mathrm{Ca} \mathrm{e}{ }^{42} \mathrm{Ca} \\
\text { na urina de } \\
72 \text { horas }\end{array}$ & $\begin{array}{l}\text { Maior no grupo tratamento } \\
(\mathrm{M}=27,3, \mathrm{DP}=4,2 \%) \text { em } \\
\text { relação ao controle } \\
(\mathrm{M}=20,8, \mathrm{DP}=2,4 \%) \\
p<0,05\end{array}$ & $\begin{array}{l}\text { Holloway } \\
\text { et al. }{ }^{16}\end{array}$ \\
\hline
\end{tabular}

M: Média; DP: Desvio-Padrão.

diferenças de 5\% na taxa de absorção de cálcio entre os grupos; dessa forma no estudo de van den Heuvel et al. ${ }^{11}$ seriam necessários ao menos doze participantes, enquanto Griffin et al. ${ }^{13,14}$ precisavam recrutar pelo menos trinta indivíduos.
Abrams et al. ${ }^{15}$ consideraram clinicamente relevante a diferença de $6 \%$ na taxa de absorção de cálcio entre os grupos, sendo necessários, portanto, no mínimo oitenta participantes para compor a amostra total. 
Tipo de frutano e quantidade consumida

O efeito da oligofrutose na biodisponibilidade de cálcio foi testado tanto em adolescentes $^{11,13}$ como em adultos jovens ${ }^{10}$, bem como em mulheres na pós-menopausa ${ }^{12}$. Já o efeito da inulina foi analisado apenas nos adultos joven ${ }^{10}$. Enquanto, o efeito da mistura de frutanos foi avaliado em adolescentes ${ }^{13-15}$ e em mulheres menopausadas ${ }^{16}$. Em nenhum dos artigos relatados foram encontradas as informações que subsidiaram a definição da quantidade de frutanos consumida.

Em seis estudos, os pesquisadores adotaram a estratégia de fornecer embalagens contendo a porção de frutanos ou de placebo a ser consumida, de acordo com o fracionamento diário pré-determinado. A partir da devolução das embalagens, os pesquisadores realizaram o controle da adesão à intervenção $0^{11,13-15}$. Não foram relatadas relações entre a adesão ao tratamento e os resultados encontrados.

Os participantes foram orientados a diluir a porção de frutanos ou placebo, contida na embalagem, em suco de laranja ${ }^{10,11}$, ou em suco de laranja enriquecido com cálcio ${ }^{13-15}$, ou ainda em chá e café ${ }^{16}$, imediatamente antes do consumo. Tahiri et al. ${ }^{12}$ não informaram o veículo utilizado para o consumo de frutanos e do placebo.

\section{Tipo de controle}

Na maioria dos estudos observou-se o uso da sacarose como controle placebo ${ }^{11-14}$. Nos estudos mais recentes ${ }^{15,16}$, a maltodextrina passou a ser utilizada, uma vez que apresenta características sensoriais indistinguíves dos frutanos, servindo como um melhor controle placebo que a sacarose ${ }^{15}$. Tanto esta quanto a maltodextrina são carboidratos completamente digeridos e absorvidos no trato gastrointestinal superior e não interferem na atividade metabólica da microbiota colônica.

van den Heuvel et al. ${ }^{10}$ utilizaram uma dieta-controle. Nas duas primeiras semanas de estudo, os indivíduos consumiram em casa a dieta oferecida pelos pesquisadores, com exceção do jantar, que era consumido na unidade de pesquisa metabólica. Na última semana de cada tratamento, os indivíduos ficaram internados na unidade de pesquisa metabólica, onde faziam todas as suas refeições. Os tratamentos testados foram oferecidos como suplemento à dieta-controle.

Geralmente a unidade de pesquisa metabólica possui diferentes cômodos onde os voluntários realizam atividades rotineiras enquanto participam do estudo, consumindo apenas os alimentos preparados e porcionados na cozinha metabólica, o que permite um controle preciso da ingestão alimentar. Na unidade metabólica também se realizam diferentes testes, exames e análises, resultando em uma avaliação mais precisa sobre os efeitos bioquímicos da dieta e/ou dos constituintes dos alimentos.

\section{Delineamento do estudo e tempo de seguimento}

Observou-se que apenas Abrams et al. ${ }^{15}$ adotaram paralelismo entro os grupos, sendo este o único ensaio de longa duração (um ano de seguimento) publicado até o momento. Nos demais, foi utilizado o delineamento cruzado; apenas van den Heuvel et al. ${ }^{10}$ não informaram o período de eliminação (washout). Observa-se que tanto o tempo de intervenção como o de washout foram variáveis (Quadro 1), não tendo sido justificados em nenhum dos trabalhos.

\section{Avaliação do consumo dietético de cálcio e vitamina D}

van Den Heuvel et al. ${ }^{11}$, embora citem que os participantes mantiveram a dieta habitual, não investigaram o consumo alimentar durante todo o período de estudo.

Ao início do ensaio, Griffin et al..$^{13}$ avaliaram o consumo alimentar por meio de questionário de frequência alimentar e forneceram 
orientação dietética para que as adolescentes atingissem e mantivessem o consumo de cálcio entre $1200 \mathrm{mg} / \mathrm{dia}$ e $1300 \mathrm{mg} / \mathrm{dia}$ durante todo o período de estudo. O acompanhamento do consumo alimentar foi realizado ao final de cada período de intervenção, por meio dos métodos de pesagem dos alimentos e registro alimentar de três dias, contemplando dois dias da semana e um dia do final de semana. Não foram observadas diferenças de consumo de cálcio entre os grupos durante as fases de estudo: grupo-tratamento com oligofrutose (Média (M)=1 524, Desvio-Padrão (DP) $=265 \mathrm{mg} / \mathrm{dia}$ ) em relação ao grupo-controle $(M=1611, D P=326 \mathrm{mg} / \mathrm{dia})(p=0,34)$, e grupo-tratamento com mistura de frutanos ( $M=1525$, $\mathrm{DP}=282 \mathrm{mg} / \mathrm{dia}$ ) em relação ao grupo-controle $(M=1495, D P=280 \mathrm{mg} / \mathrm{dia})(p=0,50)$. No estudo posterior Griffin et al..$^{14}$ citam o uso de diário alimentar, mas os resultados não foram apresentados no artigo.

Abrams et al..$^{15}$ utilizaram o método de pesagem dos alimentos nos dias de avaliação da absorção de cálcio, quando os individuos permaneciam na unidade de pesquisa metabólica - ao inicio do estudo (baseline), após 8 semanas, e após 1 ano de seguimento. Também foram utilizados diários alimentares com registro de pesagem dos alimentos consumidos durante seis dias, além de recordatório de 24 horas realizado por contato telefônico com os participantes. Os autores não observaram diferenças no consumo de cálcio dos indivíduos durante o estudo e nem entre os grupos, sendo o consumo médio inicial de $M=907$, $\mathrm{DP}=33 \mathrm{mg} / \mathrm{dia}$; após 8 semanas, de $\mathrm{M}=959$, $\mathrm{DP}=33 \mathrm{mg} / \mathrm{dia}$; e após um ano, de $M=906$, $\mathrm{DP}=29 \mathrm{mg} / \mathrm{dia}$. Este foi o único estudo com adolescentes, no qual foi avaliado também o consumo de vitamina $D$, não sendo detectadas diferenças entre os grupos durante todo o período de acompanhamento.

No estudo realizado por van Den Heuvel et al. ${ }^{10}$ com adultos jovens, a dieta-controle contendo 955mg de cálcio/dia foi oferecida para todos os grupos com a suplementação de frutanos. Os autores não informam se controlaram o consumo alimentar.
Nas duas investigações realizadas com mulheres menopausadas foi realizado controle do consumo dietético de cálcio e vitamina D. Tahiri et al..$^{12}$ forneceram orientação nutricional às participantes com o objetivo de elas consumirem cerca de $900 \mathrm{mg}$ de cálcio/dia. A avaliação do consumo alimentar foi realizada por meio de registro alimentar de quatro dias. Durante o período compreendido entre o quinto e sétimo dia após o consumo do isótopo estável de cálcio, as participantes consumiram dietas padronizadas e o consumo alimentar foi avaliado por meio do método de pesagem dos alimentos. Os autores não observaram diferenças no consumo de cálcio e de vitamina $D$ entre os grupos durante o período de seguimento.

Holloway et al. ${ }^{16}$ realizaram uma fase de equílibrio prévio, na qual as mulheres que consumiam menos que 800mg de cálcio/dia receberam suplementação com carbonato de cálcio $(500 \mathrm{mg} /$ dia) durante as três semanas antecedentes ao início do ensaio clínico. Na etapa inicial do estudo (baseline), foi avaliada a densidade mineral óssea das participantes. Marcadores bioquímicos da remodelação óssea também foram avaliados ao início, meio e final de cada período de intervenção. O consumo alimentar foi avaliado por meio de registro alimentar de três dias, ao início e ao final de cada período (que coincidia com as 72 horas de coleta de urina). Não foram observadas diferenças de consumo de cálcio entre o grupo tratamento com frutanos ( $M=1086, D P=232 \mathrm{mg} / \mathrm{dia})$ e o controle $(M=1018, D P=251 \mathrm{mg} / \mathrm{dia})$, e nem de vitamina $D$ entre os grupos $(M=25,0$, $D P=8,4 \mathrm{mg} / \mathrm{dia}$ e $25,3, \mathrm{DP}=8,0 \mathrm{mg} / \mathrm{dia}$, respectivamente).

\section{Método de avaliação da absorção de cálcio}

Em cinco estudos foram realizadas comparações da taxa de absorção de cálcio do grupo tratamento e do grupo controle, ao final do período de intervenção. Apenas nos estudos mais recentes ${ }^{15,16}$ a avaliação da absorção de cálcio foi 
realizada ao início (baseline) e ao final do estudo, permitindo assim uma avaliação mais completa, por meio da (1) caracterização e comparação dos grupos ao início do estudo, (2) comparação entre os grupos ao final do tempo de seguimento, (3) avaliação de mudanças na taxa de absorção de cálcio em função do produto consumido e (4) comparação da magnitude do efeito entre os grupos.

No estudo de Tahiri et al. ${ }^{12}$, a biodisponibilidade do cálcio foi avaliada por meio do balanço metabólico, método que estima a diferença entre a ingestão oral e a excreção fecal de cálcio. A principal desvantagem deve-se ao fato de esse método quantificar tanto o cálcio que não foi absorvido no trato gastrointestinal como a perda fecal endógena ${ }^{17}$, de forma que o resultado refere-se à absorção aparente de cálcio.

Nos demais trabalhos, a absorção intestinal real de cálcio foi analisada. Basicamente, o procedimento metodológico envolvia a administração de dois isótopos estáveis de cálcio, um por via oral e outro por via parenteral. A absorção do cálcio é estimada a partir da recuperação relativa dos isótopos na urina, determinados por espectrometria de massas. Os isótopos utilizados em cada estudo são mencionados no Quadro 1, sendo a primeira citação referente ao isótopo administrado por via oral e a segunda ao isótopo administrado por via intravenosa. O tempo de coleta de urina foi variável entre os estudos (de 24 a 72 horas).

\section{Efeito dos frutanos na absorção de cálcio}

Após 9 dias de consumo de $15 \mathrm{~g}$ de oligofrutose, houve aumento da absorção de cálcio dos meninos ${ }^{11}$. O consumo de $8 \mathrm{~g}$ de oligofrutose durante 21 dias não alterou a absorção de cálcio de meninas, enquanto o consumo da mistura de frutanos aumentou essa taxa entre as meninas pré-puberes e púberes ${ }^{13,14}$; de outro lado, não foram observadas diferenças no consumo dietético nem na excreção urinária de cálcio entre os grupos $^{13}$. O consumo diário de $8 \mathrm{~g}$ da mistura de frutanos durante um período de 2 a 12 meses resultou em aumento da absorção de cálcio de meninos e meninas pré-púberes ${ }^{15}$. Ao comparar os resultados do início (baseline) e do final do período de seguimento, os autores observaram que o consumo de frutanos durante dois meses resultou em aumento da absorção de cálcio em $M=8,5, D P=1,6 \%$ em relação ao controle $(p<0,001)$, e durante um ano esse aumento foi de $\mathrm{M}=5,9, \mathrm{DP}=12,8 \%(p=0,04)$. Não foram observadas diferenças no consumo dietético nem na excreção urinária de cálcio entre os grupos $(p>0,2)^{15}$.

O consumo diário de $15 \mathrm{~g}$ de inulina ou de oligofrutose durante 21 dias não alterou a taxa de absorção de cálcio de homens jovens ${ }^{10}$. Não houve alteração na absorção intestinal de cálcio de mulheres menopausadas que consumiram diariamente $10 \mathrm{~g}$ de oligofrutose durante 5 semanas $^{12}$. Os autores também não observaram diferenças no consumo dietético nem na excreção fecal e urinária desse mineral entre os grupos. Também não foram encontradas diferenças nos marcadores bioquímicos da remodelação óssea. Em outro estudo, após 6 semanas de consumo diário de $10 \mathrm{~g}$ da mistura de frutanos, houve aumento da absorção de cálcio de mulheres na pós-menopausa ${ }^{16}$. Em relação ao baseline, o consumo de frutanos ocasionou aumento $(M=+5,1$, $\mathrm{DP}=2,1 \%, p<0,05)$ e o placebo ocasionou redução ( $M=-3,3, \mathrm{DP}=2,2 \%, p<0,05)$ da taxa de absorção de cálcio. Os autores observaram que as mulheres que apresentavam menor densidade mineral óssea foram as que apresentaram maior aumento da taxa de absorção de cálcio após consumirem a mistura de frutanos ${ }^{16}$.

\section{DISCUSS Ã O}

\section{Ensaios clínicos de curta duração}

O número limitado de artigos relatados e as diferenças relativas ao planejamento experimental, tempo de estudo, tipo de frutano e quantidade consumida, faixa etária dos indivíduos e 
método utilizado para a avaliação da taxa de absorção de cálcio impedem a generalização dos resultados observados.

Possivelmente, no ensaio de van Den Heuvel et al. ${ }^{10}$ não foi observado efeito do consumo de frutanos na taxa de absorção de cálcio, devido à limitação do método de coleta de urina por 24 horas, período que não permite avaliar a absorção colônica de cálcio. Observa-se ampliação do tempo de coleta de urina nos demais estudos, com variação de 36 a 72 horas (Quadro 1).

A presença de indivíduos em diferentes estágios hormonais pode ser um fator responsável pela falta de efeito no estudo de Thairi et al. ${ }^{12}$. Quando as mulheres foram estratificadas por tempo de pós-menopausa, os autores observaram uma tendência de aumento da absorção de cálcio naquelas com mais de seis anos nessa fase. Foram observados resultados positivos no estudo de Holloway et al. ${ }^{16}$, que investigaram apenas mulheres que estavam na pós-menopausa por pelo menos uma década.

Além disso, a inulina e a oligofrutose, bem como a sua mistura, ainda não foram testadas sob as mesmas condições experimentais, o que permite apenas lançar hipóteses sobre um possível efeito sinérgico entre elas, considerando os resultados obtidos em modelos animais ${ }^{8,9}$. No entanto, as evidências científicas sugerem que o consumo diário da mistura pode aumentar a taxa de absorção de cálcio de adolescentes ${ }^{13-15}$ e de mulheres na pós-menopausa ${ }^{16}$.

\section{Ensaio clínico de longa duração}

Abrams et al..$^{15}$ realizaram o único estudo de longa duração publicado até o momento. A absorção de cálcio foi significativamente maior no grupo que consumiu os frutanos durante um ano de estudo (diferença: $M=5,9, D P=2,8 \%$ ), como mostra o Quadro 1, resultando em maior agregação de cálcio no esqueleto para o grupo tratamento $(M=218, D P=10 \mathrm{mg} / \mathrm{dia})$ do que no controle $(M=189, D P=10 \mathrm{mg} / \mathrm{dia}), p=0,04$. Tam- bém houve aumento significativo na densidade mineral óssea corpórea ( $\mathrm{M}=0,047, \mathrm{DP}=0,004 \mathrm{~g} /$ $\mathrm{cm}^{2} /$ ano versus $\left.\mathrm{M}=0,032, \mathrm{DP}=0,004 \mathrm{~g} / \mathrm{cm}^{2} / \mathrm{ano}\right)$, $p=0,01$.

Posteriormente, os autores investigaram os dados dos 48 adolescentes que fizeram parte do grupo tratamento ${ }^{18}$. Destes, 32 (67\%) foram considerados sensíveis à intervenção com frutanos, ou seja, apresentaram aumento de pelo menos 3\% da absorção de cálcio após 8 semanas de estudo. Os autores estimaram que $15 \mathrm{~g}$ adicionais de cálcio podem ser depositados no esqueleto desses indivíduos. Essa quantidade representa cerca de $10-15 \%$ da taxa anual de cálcio que é depositada nos ossos, nessa faixa etária. $O$ benefício nos indivíduos sensíveis à intervenção seria comparável a aumentar a ingestão dietética de cálcio em 250-320mg/dia. A sensibilidade à intervenção pode ser influenciada por fatores genéticos, pelo consumo usual de frutanos, por outros aspectos relacionados à dieta, pela adesão à intervenção, dentre outros fatores ainda não identificados $^{18 ;}$; por isso, ainda não é possível avaliar a significância desses incrementos para a saúde dos indivíduos.

Abrams et al. ${ }^{19}$ realizaram um novo estudo com 13 adultos jovens (18-27 anos), que consumiram $8 \mathrm{~g} /$ dia de inulina e oligofrutose durante oito semanas. Oito $(62,0 \%)$ indivíduos responderam à intervenção (aumento de 3,0\% da absorção de cálcio), sendo submetidos a um teste para avaliar a cinética de absorção de cálcio durante 26 horas. Considerando que a absorção colônica de cálcio se inicia 7 horas após a ingestão, os autores concluíram que a absorção colônica é responsável por $M=69,6, D P=18,6 \%$ do aumento da absorção de cálcio ocasionada pelo consumo dos frutanos, fortalecendo as hipóteses elaboradas com base nos modelos animais.

\section{Direcionamentos para investigações futuras}

A revisão sistemática dos sete artigos selecionados aponta a evolução do conhecimento na 
temática investigada. Os estudos iniciais indicaram melhores resultados após o consumo da mistura de frutanos ${ }^{13,14}$, a qual passou a ser investigada com maior profundidade em um estudo de longa duração, com maior número de participantes e maior controle da ingestão alimentar, possibilitando a avaliação da mineralização óssea ${ }^{15}$. Observam-se também mudanças em relação aos critérios de seleção dos indivíduos, com a padronização do estágio fisiológico; à escolha do placebo, com aspecto e características sensoriais mais parecidas com as dos frutanos; ao controle do consumo dietético de cálcio e vitamina $\mathrm{D}^{15,16}$; à investigação de fatores que podem estar relacionados ao metabolismo de cálcio, como o polimorfismo genético ${ }^{15}$, a cinética de absorção de cálcio ${ }^{19}$ e marcadores da remodelação óssea ${ }^{16}$, na tentativa de entender os mecanismos envolvidos, que ainda não foram completamente elucidados.

Esta revisão permitiu elaborar sugestões para investigações futuras. Há necessidade de se realizarem mais estudos no sentido de (1) fornecer comprovação científica a respeito do possível efeito sinérgico da inulina e da oligofrutose em potencializar a absorção intestinal de cálcio; (2) determinar a quantidade e o tempo mínimos de consumo de frutanos, necessários para o aumento da absorção de cálcio e da mineralização óssea de indivíduos em diferentes faixas etárias; (3) esclarecer os mecanismos envolvidos, visando favorecer também indivíduos não saudáveis.

A utilização de ensaios cruzados é uma boa estratégia para avaliação do efeito do consumo de frutanos na absorção de cálcio, uma vez que os indivíduos são os seus próprios controles. No entanto, especial atenção deve ser dada ao período de eliminação (washout). Ainda não se sabe qual o tempo ideal de descontinuação do consumo de frutanos para evitar efeitos carreadores. Para estudos com maior tempo de seguimento, o delineamento cruzado torna-se impraticável.

Até o momento, apenas um único estudo possibilitou avaliar o efeito sobre a mineralização óssea ${ }^{15}$. Considerando os cuidados adotados quanto ao delineamento de estudo, a avaliação e controle de possíveis confundidores, bem como a baixa taxa de perdas ( 6\%) durante os 12 meses de seguimento, este seria um ensaio modelo para ser adotado em futuras investigações.

Há necessidade de estudos controlados de longa duração, de tamanho amostral com maior poder de teste, para avaliar um possível efeito dos frutanos na mineralização óssea de mulheres na pós-menopausa, grupo populacional que pode se beneficiar dos potenciais efeitos dos frutanos. Seria interessante também comparar os frutanos aos sais de cálcio tradicionalmente utilizados, ou ainda pesquisar se o consumo associado poderia potencializar os efeitos da suplementação mineral e contribuir para maior manutenção da mineralização óssea nesse grupo populacional.

Deve-se considerar o fato de que apenas indivíduos com consumo de cálcio próximo ou superior ao recomendado para a faixa etária participaram dos estudos (Quadro 1), e que ainda não se conhecem os efeitos do consumo de frutanos em indivíduos com baixo consumo desse mineral. Além disso, a sensibilidade à intervenção pode ser influenciada por fatores genéticos, pelo consumo usual de frutanos e outros aspectos relacionados à dieta, e pela adesão à intervenção, dentre outros fatores ainda não identificados ${ }^{18}$.

Também é necessário conhecer os possíveis efeitos colaterais da suplementação com frutanos. De acordo com Abrams et al. ${ }^{15}$, o consumo diário de $8 \mathrm{~g}$ da mistura de frutanos parece ser seguro, uma vez que apenas um indivíduo $(\sim 2 \%)$ reportou desconforto, com sintomas gastrointestinais que se resolveram rapidamente quando o mesmo foi suspenso.

As evidências científicas ${ }^{6-9,19}$ sugerem que a absorção colônica é majoritariamente responsável pelo aumento da absorção de cálcio ocasionada pelo consumo dos frutanos. Assim, o consumo de frutanos pode ser especialmente importante em condições fisiológicas ou anatômicas em que a absorção de cálcio no intestino delgado está prejudicada. Este seria um tópico 
muito importante para investigações futuras, uma vez que, teoricamente, os frutanos poderiam favorecer de forma especial a saúde desses indivíduos.

Novos estudos são necessários para investigar o efeito do consumo de frutanos no aumento da absorção de cálcio e da massa óssea de indivíduos saudáveis, verificando se tal efeito se restringiria a determinada faixa etária ou estado fisiológico, bem como se a sensibilidade à intervenção seria condicionada por fatores dietéticos e genéticos.

\section{CONSIDERAÇÕ ES FIN A IS}

Devido à sua fundamental importância para a saúde, a necessidade de adequação da ingestão do cálcio tem despertado a atenção de inúmeras pesquisas e também de políticas de Saúde Pública. Frequentemente tem-se observado a disparidade entre a quantidade recomendada e a tipicamente consumida pela população de forma geral. Assim como o consumo dietético, a absorção intestinal é um fator crucial para a saúde óssea. Portanto, estratégias para aumentar o consumo e a absorção de cálcio da dieta são consideradas relevantes.

Nesse contexto, os frutanos do tipo inulina podem ter especial importância. Resultados de quatro dos sete ensaios clínicos duplo-cegos controlados aleatorizados, incluídos neste trabalho, indicam que o consumo diário da mistura de frutanos aumenta a absorção de cálcio, dependendo da idade cronológica e fisiológica, e do estado menopausal de indivíduos saudáveis que consomem quantidades adequadas de cálcio.

Essa revisão demonstra a necessidade de mais ensaios clínicos de longa duração, nos quais tanto a absorção de cálcio como a densidade mineral óssea sejam avaliadas. Futuros estudos devem contribuir para a compreensão dos mecanismos de ação dos frutanos no aumento da absorção de cálcio; para avaliar se esse efeito persiste em longo prazo e se pode ser considerado como benefício real para a saúde óssea; para testar se tais efeitos poderiam beneficiar indivíduos de outras faixas etárias e diferentes condições fisiológicas.

No futuro, evidências científicas consistentes poderão indicar a suplementação com frutanos como uma estratégia de prevenção da osteoporose; poderão também direcionar o desenvolvimento de produtos que contribuam para o aumento da absorção de cálcio e da mineralização óssea.

\section{COLABORADORES}

VD CAPRILES realizou o levantamento bibliográfico, analisou os dados e redigiu o manuscrito. JAG ARÊAS realizou a orientação do trabalho e a revisão do manuscrito.

\section{A GRADECIMENTOS}

À Fundação de Amparo à Pesquisa do Estado de São Paulo, pela concessão da bolsa de doutorado direto, processo 04/14127-3.

\section{REFER Ê NCIAS}

1. Roberfroid M, Delzenne N. Dietary fructans. Annu Rev Nutr. 1998; 18(1):117-43.

2. Roberfroid M. Introducing inulin-type fructans. $\mathrm{Br}$ J Nutr. 2005; 93(Suppl. 1):S13-25.

3. Franck A. Technological functionality of inulin and oligofructose. Br J Nutr. 2002; 87(Suppl. 2):S287-91.

4. Gibson G, Probert H, Loo J, Rastall R, Roberfroid $M$. Dietary modulation of the human colonic microbiota: updating the concept of prebiotics. Nutr Res Rev. 2004; 17(2):259-75.

5. Kolida S, Gibson G. Prebiotic capacity of inulintype fructans. J Nutr. 2007; 137(11 Suppl.):2503S-6S.

6. Scholz-Ahrens K, Schrezenmeir J. Inulin and oligofructose and mineral metabolism: the evidence from animal trials. J Nutr. 2007; 137(11 Suppl.): 2513S-23S.

7. Scholz-Ahrens K, Schrezenmeir J. Inulin, oligofructose and mineral metabolism: experimental data and mechanism. Br J Nutr. 2002; 87(Suppl. 2):S179-S86.

8. Coudray C, Tressol J, Gueux E, Rayssiguier Y. Effects of inulin-type fructans of different chain length and 
type of branching on intestinal absorption and balance of calcium and magnesium in rats. Eur J Nutr. 2003; 42(2):91-8.

9. van Loo J. The specificity of the interaction with intestinal bacterial fermentation by prebiotics determines their physiological efficacy. Nutr Res Rev. 2004; 17(1):89-98.

10. van den Heuvel E, Schaafsma G, Muys T, van Dokkum W. Nondigestible oligosaccharides do not interfere with calcium and nonheme-iron absorption in young, healthy men. Am J Clin Nutr. 1998; 67(3):445-51.

11.van den Heuvel E, Muys T, van Dokkum W, Schaafsma G. Oligofructose stimulates calcium absorption in adolescents. Am J Clin Nutr. 1999; 69(3):544-8.

12. Tahiri M, Tressol J, Arnaud Y, Bornet F, BouteloupDemange C, Feillet-Coudray C, et al. Effect of shortchain fructooligosaccharides on intestinal calcium absorption and calcium status in postmenopausal women: a stable-isotope study. Am J Clin Nutr. 2003; 77(2):449-57.

13. Griffin I, Davila P, Abrams S. Non-digestible oligosaccharides and calcium absorption in girls with adequate calcium intakes. Br J Nutr. 2002; 87(Suppl. 2):S187-91.

14. Griffin I, Hicks P, Heaney R, Abrams S. Enriched chicory inulin increases calcium absorption mainly in girls with lower calcium absorption. Nutr Res. 2003; 23(7):901-9.
15. Abrams S, Griffin I, Hawthorne K, Liang L, Gunn S, Darlington $\mathrm{G}$, et al. Combination of prebiotic shortand long-chain inulin-type fructans enhances calcium absorption and bone mineralization in young adolescents. Am J Clin Nutr. 2005; 82(2): 471-6.

16. Holloway L, Moynihan S, Abrams S, Kent K, Hsu A, Friedlander A. Effects of oligofructose-enriched inulin on intestinal absorption of calcium and magnesium and bone turnover markers in postmenopausal women. Br J Nutr. 2007; 97(2): 365-72.

17. Pereira GAP, Genaro OS, Pinheiro MM, Szejnfeld VL, Martini LA. Cálcio dietético: estratégias para otimizar o consumo. Rev Bras Reumatol. 2009; 49(2):164-80.

18. Abrams S, Griffin I, Hawthorne K. Young adolescents who respond to an inulin-type fructan substantially increase total absorbed calcium and daily calcium accretion to the skeleton. J Nutr. 2007; (11 Suppl.): 2524S-6S

19. Abrams S, Hawthorne K, Aliu O, Hicks P, Chen Z, Griffin I. An inulin-type fructan enhances calcium absorption primarily via an effect on colonic absorption in humans. J Nutr. 2007; 137(10): 2208-12.

Recebido em: 2/8/2010

Versão final reapresentada em: 27/4/2011 Aprovado em: 24/8/2011 
